Julio César Alonso*

Diego Alexánder Martínez Quintero*"

Universidad ICESI, Cali, Colombia

Recibido: 16 de septiembre de 2016 Concepto de evaluación: 14 de febrero de 2017

Aprobado: 25 de mayo de 2017

Artículo de investigación (C) 2017 Universidad Católica de Colombia. Facultad de Ciencias Económicas y Administrativas. Todos los derechos reservados

* Ph.D. in Economics, M.Sc. in Economics, M.Sc. in Statistics, Economista. Docente-investigador del Departamento de Economía, Facultad de Ciencias Administrativas y Económicas, Universidad ICESI, Cali, Colombia. Dirección de correspondencia: Calle 18 \# 122-135,

Cali, Colombia. Correo Electrónico: jcalonso@icesi.edu.co

** Magíster en Economía, Economista. Docente-investigador del Departamento de Economía, Facultad de Ciencias Administrativas y Económicas, Universidad ICESI, Cali, Colombia. Dirección de correspondencia: Calle 18 no 122-135

Cali, Colombia. Correo Electrónico: diegomartinez91@gmail.com

\section{Impacto del precio del petróleo sobre el PIB de los países de la Alianza del Pacífico*}

\section{RESUMEN}

Este artículo busca determinar el efecto del precio del petróleo sobre el producto interno bruto (PIB) en cada uno de los países que integran la Alianza del Pacífico (AP). Se emplea un modelo VAR estructural y datos trimestrales que permiten concluir que existe una relación de largo plazo entre el precio internacional del petróleo y el PIB en cada uno de los países miembro de la AP. Se encuentra que un aumento inesperado en los precios del petróleo no afecta el nivel de actividad económica en Perú. Para Chile, un importador neto de petróleo, el efecto de choque similar genera un aumento en el PIB, contrario a lo que se esperaría teóricamente. Para México y Colombia, exportadores netos de petróleo, el efecto de un choque positivo inesperado en el precio de petróleo es positivo sobre el PIB: en Colombia, el efecto positivo se observa para los primeros seis trimestres, mientras que para México solo está presente en el segundo y tercer trimestre. La heterogeneidad en la respuesta de estos países a choques en el precio del petróleo muestra los grandes retos que implicará la coordinación de la política económica entre los países miembro de la AP.

Palabras clave: Alianza del Pacífico, precio del petróleo, PIB, series de tiempo, VAR.

JEL: C20, C22, E30, F43, N16

\section{Oil prices impact on the GDP of the Pacific Alliance Countries}

\section{ABSTRACT}

This article seeks to determine the effect of oil prices on gross domestic product (GDP) in the member countries of the Pacific Alliance (PA). A structural VAR model and quarterly data are used, which allow concluding that there is a long-term relationship between the international price of oil and GDP in each of the PA member countries. It was found that an unexpected increase in oil prices does not affect the level of economic activity in Peru. For Chile, which is a net importer of oil, a similar shock effect increases GDP, contrary to what is expected in theory. For Mexico and Colombia, net exporters of oil, an unexpected positive oil price shock positively affects GDP; in Colombia,

Este artículo fue desarrollado como parte de la agenda de investigación del Programa de Estudios de la Alianza del Pacífico (PEAP) de la Universidad Icesi. Los autores forman parte del grupo de investigación Economía, Políticas Públicas y Métodos Cuantitativos de esta misma universidad. 
the positive effect is observed in the first six quarters, while for Mexico it is only present in the second and third quarters. These countries' heterogeneous responses to oil price shocks are evidence of the many challenges that economic policy coordination among PA member countries has to face.

Keywords: Pacific Alliance, oil price, GDP, time series, VAR.

\section{Impacto do preço do petróleo sobre o PIB dos países- da Aliança do Pacífico}

\section{RESUMO}

Este artigo busca determinar o efeito do preço do petróleo sobre o produto interno bruto (PIB) em cada um dos países que integram a Aliança do Pacífico (AP). Emprega-se um modelo VAR estrutural e dados trimestrais que permitem concluir que existe uma relação de longo prazo entre o preço internacional do petróleo e o PIB em cada um dos países membros da AP. Constata-se que um aumento inesperado no preço do petróleo não afeta o nível de atividade econômica no Peru. Para o Chile, um importador líquido de petróleo, o efeito de choque similar gera um aumento no PIB, contrário ao que seria esperado teoricamente. Para o México e a Colômbia, exportadores líquidos de petróleo, o efeito de um choque positivo inesperado no preço do petróleo é positivo sobre o PIB: na Colômbia, o efeito positivo é observado para os primeiros seis trimestres, enquanto para o México só está presente no segundo e terceiro trimestre. A heterogeneidade na resposta destes países a choques no preço do petróleo mostra os grandes desafios que implicará a coordenação da política econômica entre os países membros da AP.

Palavras-chave: Aliança do Pacífico, PIB, preço do petróleo, séries de tempo, VAR. 


\section{INTRODUCCIÓN}

Determinar el efecto del precio del petróleo sobre el comportamiento de una economía ha sido de gran interés tanto para hacedores de políticas públicas como para investigadores. Este interés es motivado por el hecho de que el petróleo fue la principal fuente de energía en el siglo pasado e inicios de este. Autores como He, Wang y Lai (2010), Basnet y Upadhyaya (2015) y Denis y Etornam (2015) reconocen que esta importancia se incrementó significativamente después de la Segunda Guerra Mundial con el aumento del uso del automóvil como principal medio de transporte. De hecho, Denis y Etornam (2015) sugieren que el petróleo es una fuente de energía esencial, un irremplazable combustible para el transporte y una materia prima indispensable en muchos procesos de manufactura. Esto hace que cualquier movimiento en el precio de esta materia prima implique movimientos en los agregados económicos.

Zaidi, Karim y Azman-Saini (2013) afirman que entender los vínculos entre los choques extranjeros del precio del petróleo con las variables macroeconómicas es crucial a la hora de implementar políticas adecuadas que propendan a mantener la estabilidad económica en los países.

En los últimos años, el precio del petróleo ha experimentado una gran volatilidad. En 1998 se observó un precio mínimo de 12,9 dólares por barril; pasó luego a un precio máximo de 123,9 dólares por barril en 2008; por su parte, en 2009 este precio se ubicó en 43,1 dólares por barril; para 2013 se observó un precio máximo de 105,8 dólares por barril; y luego, en 2015, se ubicó en un precio mínimo de 41,9 dólares por barril ${ }^{1}$. Estas variaciones han generado grandes sobresaltos en las economías de países emergentes.

Por otro lado, la naciente Alianza del Pacífico (AP) ha sido concebida como un mecanismo de integración económica-comercial conformado por Chile, Colombia, México y Perú con miras al mercado asiático. Esta alianza implicará armonizar las

1 Datos obtenidos de Federal Reserve Bank of St. Louis, FRED Economic Data. instituciones económicas y políticas de los cuatro países y la coordinación de las políticas macroeconómicas de estos. No obstante, poco se conoce aún del comportamiento de estos países frente a choques internacionales. Así, es importante estudiar el efecto de los cambios en el precio del petróleo sobre cada una de las economías de la AP. Esto permitiría entender las similitudes o diferencias que presentan las reacciones de las economías de los países miembro de la AP frente a choques en el precio del petróleo y, por tanto, las implicaciones de política macroeconómica para cada uno de ellos.

De acuerdo con la discusión de autores como Jiménez-Rodríguez y Sánchez (2005), a priori se esperaría que el efecto no fuera igual en los países miembro de la AP, debido a la condición de México y Colombia de exportadores netos de petróleo y la de importadores netos de Chile y Perú. El propósito del presente estudio es dar un primer paso en el estudio del efecto de los cambios en el precio del petróleo sobre los principales agregados macroeconómicos. Así, el objetivo será estimar el efecto de los cambios en el precio del petróleo sobre el PIB de cada uno de los países de la AP.

En la literatura se encuentran varios mecanismos de transmisión a través de los cuales los cambios en el precio del petróleo afectan la actividad económica. Estos incluyen mecanismos que se activan tanto por el lado de la oferta agregada como por el de la demanda agregada. Por el lado de esta última, el impacto de las variaciones en el precio del petróleo se deriva del efecto sobre el consumo y la inversión (para una discusión más amplia de este tema, véase: Mork y Hall, 1980; Brown y Yücel, 2002; Lardic y Mignon, 2008; Jiménez-Rodríguez y Sánchez, 2005). Las fluctuaciones en el precio del petróleo influencian de manera directa los patrones de consumo y gastos de los hogares. El argumento común en la literatura es que, dado que la elasticidad del precio de la demanda por petróleo es generalmente baja, precios de petróleo más altos pueden forzar a los hogares a recortar sus gastos en otros bienes y servicios.

En este sentido, Sill (2007) sugiere que mayores precios del petróleo pueden reducir la 
demanda agregada por otros bienes, porque ellos reducen la riqueza $y$, al mismo tiempo, se induce incertidumbre sobre el futuro. Por su parte, Fernald y Trehan (2005) argumentan que mayores precios del petróleo funcionan como un incremento en los impuestos sobre el consumidor, porque el pago extra que hace el consumidor a los productores de petróleo extranjeros ya no puede ser gastado en otra clase de bienes de consumo. Del mismo modo, Bernanke (1983) encuentra que mayores precios del petróleo tendrán un efecto adverso sobre el desempeño económico en el corto plazo, precisamente debido a su habilidad para alterar las compras de una gran lista de bienes de consumo e inversión.

Por el lado de la oferta agregada, el efecto está asociado con los costos de producción. Así, un incremento en el precio del petróleo aumenta los costos de producción, porque el crudo de petróleo es un insumo importante en los procesos productivos. Además, el incremento en el precio del petróleo aumenta los costos de distribución y transporte de los demás bienes y servicios. Adicionalmente, los precios del petróleo afectan indirectamente los costos de manufactura. De esta manera, incrementos en los costos de producción conllevan una disminución en la producción y, por último, una disminución en la oferta agregada de la economía (Basnet y Upadhyaya, 2015; Cunado y De Gracia, 2005; Hamilton, 1983, 2003; JiménezRodríguez y Sánchez, 2005; Mork y Hall, 1980; Zhang y Qianqian, 2011).

Denis y Etornam (2015) argumentan que la literatura empírica que estudia el efecto de los cambios del precio del petróleo sobre las variables macroeconómicas es extensa en economías desarrolladas y relativamente escasa para el caso de las economías emergentes. Autores como Hamilton (1996, 2003), Hooker (1996, 1999) y Huntington (1998) estudian esta relación. En el artículo seminal de Hamilton (1983), se documenta una relación sistemática y significativa entre el precio del petróleo y el nivel de actividad económica de Estados Unidos. Incluso, este autor plantea que los cambios en el precio del petróleo han sido uno de los principales causantes de 9 de las 10 recesiones de Estados Unidos ocurridas después de la Segunda Guerra Mundial hasta 1983.

Similares hallazgos en torno el impacto del precio del petróleo sobre las variables macroeconómicas se encuentran en Cebula, Saadatmand y Piccone (2002), Cunado y De Gracia (2005), Cologni y Manera (2008) y Kilian (2008). Por su parte, Backus y Crucini (2000) muestran que una gran parte de la variabilidad en los términos de intercambio está asociada con movimientos extremos en el precio del petróleo, hecho que se refleja finalmente sobre el comportamiento de la demanda agregada. Jiménez-Rodríguez y Sánchez (2005) evalúan el efecto de los cambios en el precio del petróleo sobre el nivel de actividad económica real de los principales países industrializados (una mezcla de países exportadores e importadores). Estos hallazgos sugieren que el incremento en la volatilidad, en los términos de intercambio desde el sistema de Bretton Woods, parece que fue generado en gran parte por el incremento en las fluctuaciones del precio del petróleo, más que por la volatilidad de la tasa de cambio real o nominal (TCR). Además, sus hallazgos concluyen que la tasa de crecimiento del producto interno bruto (PIB) real en países importadores de petróleo es afectado negativamente por incrementos en el precio del petróleo.

En el caso de los países de la AP, la literatura es incipiente. Es posible encontrar algunos autores que estudian la relación precio-petróleo y variables macroeconómicas, como Pedersen y Ricaurte (2013) para el caso de Chile. Para Perú, Gallardo, Vásquez-Cordano y Bendezú (2005) evidencian que la volatilidad del precio del petróleo en el corto y largo plazo se trasmite a las economías a través del canal de las importaciones. En el caso de Colombia, Perilla (2010) muestra una relación positiva entre los cambios de los precios del petróleo y el crecimiento económico. Para México, Alarco (2006) encuentra que mayores precios reales del petróleo se asocian con mayores tasas de crecimiento de la producción, mientras que las contracciones se relacionan con caídas en el producto mexicano. 
De esta manera, el propósito del presente estudio es estimar y comparar el efecto de los cambios en el precio del petróleo sobre el PIB de cada uno de los países de la AP: Chile, Colombia, México y Perú, usando series de datos trimestrales de 1980Q2 a 2014Q3 para el caso de Chile y México, 1990Q1 a 2014Q3 para Perú y 1994Q1 a 2014Q3 para Colombia. En otras palabras, este estudio busca responder a dos preguntas: a) ¿cuál es el efecto sobre el PIB de un incremento o una caída en el precio internacional del petróleo para cada país miembro de la AP?; b) ies este efecto similar o no entre estos países?

La respuesta a estas preguntas tiene implicaciones de política para los países que integran la AP; en especial, permitirá empezar a reflexionar sobre los retos que implicará la coordinación de políticas macroeconómicas entre los cuatro países. Las respuestas a estas preguntas también son de interés para los empresarios de la AP o para aquellos interesados en invertir en los países de la AP. Al conocer cómo responde el nivel de actividad de estos países, los empresarios podrían responder mejor y adaptarse a los cambios en el entorno, fruto de los cambios en los precios del petróleo.

Además de la presente introducción, el artículo se organiza de la siguiente forma: inicialmente, se presentan la metodología y los datos usados en el estudio; luego se muestran los resultados empíricos y la discusión; finalmente, se presentan el resumen, las conclusiones y las implicaciones de política.

\section{METODOLOGÍA Y DATOS}

Como se indicó, este artículo busca estimar el impacto de los cambios en el precio del petróleo sobre el PIB real de cada uno de los países que integran la AP. La discusión de los mecanismos de transmisión del precio del petróleo sobre la producción se esbozaron en la introducción, pero un análisis más amplio se puede encontrar en Mork y Hall (1980), Brown y Yücel (2002), Lardic y Mignon (2008), JiménezRodríguez y Sánchez (2005), Cunado y De Gracia (2005), Hamilton (2003) y Zhang y Qianqian (2011).
Para estimar el efecto de una fluctuación del precio del petróleo sobre el PIB, se empleará un modelo VAR estructural (SVAR, por su sigla en inglés), como lo sugieren Basnet y Upadhyaya (2015). Las variables incluidas en el VAR son el logaritmo del precio del petróleo (Lppetroleo $)_{t}$ y el logaritmo del PIB real (Lrpib $\left.{ }_{t}\right)$, y como variables de control se emplean en el VAR el índice de precio del consumidor $\left(\operatorname{Lipc}_{t}\right)$ y la tasa de cambio real $\left(\right.$ Ltcr $\left._{t}\right)$.

De acuerdo con Basnet y Upadhyaya (2015), el modelo VAR estructural que se empleará en este estudio es:

$$
\begin{aligned}
& \text { A0Xt }=A 1 X t-1+\ldots+\text { ApXt }-p+\varepsilon t \\
& \text { Donde: } \\
& X t=\left[\text { Lppetroleo }_{t^{\prime}} \text { Lrpib }_{t^{\prime}} \text { Lipc }_{t^{\prime}} \text { Ltcr }_{t}\right] .
\end{aligned}
$$

$A_{i}$ : matriz de $4 \times 4$ de coeficientes para $i=0,1, \ldots, P$.

$\varepsilon_{t}=\left[\varepsilon^{L p p e t r o l e o}, \varepsilon^{L r p i b}, \varepsilon^{L i p c}, \varepsilon^{L t c r}\right]^{t}$ : vector de errores estructural (iid).

La matriz de coeficientes de la ecuación estructural puede ser obtenida estimando la forma reducida:

$$
\begin{aligned}
& A 0 X t=A 1 X t-1+\ldots+A p X t-p+\varepsilon t \\
& X t=A^{-1} A 1 X t-1+\ldots+A 0^{-1} A p X t-p+A 0^{-1} \varepsilon t \\
& X t=B(L) X t+\mu t
\end{aligned}
$$

Donde $B(L) X t=A_{0}^{-1} A_{1}(L)$ con $A_{0} \mu_{t}=\varepsilon_{t^{*}}$. El residuo $\mu_{t}$ es la forma reducida del VAR y se asume que es iid también. $A 1(L)$ es una matriz de polinomios en el operador de rezagos (para una explicación detallada, ver Enders, 2004; Park, Chung y Lee, 2011).

\section{RESTRICCIONES CONTEMPORÁNEAS Y DE IDENTIFICACIÓN}

Las restricciones de identificación que se emplearán están basadas en diferentes teorías e intuiciones económicas. En este análisis se utilizan solo restricciones de corto plazo sobre las relaciones contemporáneas, porque, desde la perspectiva de Christiano, Eichenbaum y Vigfusson (2007), 
el modelo VAR estructural basado en restricciones de corto plazo tiene un mejor desempeño. Las restricciones de corto plazo principalmente controlan los efectos de retroalimentación entre las variables en el modelo.

La identificación en la ecuación [2] es obtenida imponiendo restricciones sobre las relaciones contemporáneas entre las variables incluidas en el VAR estructural:

$$
\begin{aligned}
& {\left[\begin{array}{cccc}
1 & 0 & 0 & 0 \\
a_{21} & 1 & 0 & 0 \\
a_{31} & a_{32} & 1 & 0 \\
a_{41} & a_{42} & a_{43} & 1
\end{array}\right]\left[\begin{array}{c}
\mu_{\text {Lppetroleo }} \\
\mu_{\text {Lrpib }} \\
\mu_{\text {Lipc }} \\
\mu_{\text {Ltcr }}
\end{array}\right]=} \\
& {\left[\begin{array}{llll}
1 & 0 & 0 & 0 \\
0 & 1 & 0 & 0 \\
0 & 0 & 1 & 0 \\
0 & 0 & 0 & 1
\end{array}\right]\left[\begin{array}{c}
\varepsilon_{\text {Lppetroleo }} \\
\varepsilon_{\text {Lrpib }} \\
\varepsilon_{\text {Lipc }} \\
\varepsilon_{\text {Ltcr }}
\end{array}\right]}
\end{aligned}
$$

Las restricciones de identificación están soportadas en varias razones económicas. En primer lugar, cada uno de los países de la AP son pequeñas economías abiertas, tomadoras de precios en el mercado de petróleo. Así como el precio del petróleo es determinado por las condiciones de la demanda y oferta global, el nivel de producción doméstica, la inflación y la tasa de cambio en cualquiera de los países de la muestra no tendrán efecto sobre dicho precio. De esta manera, se asume que el precio del petróleo es exógeno. Entonces, el término error de la forma reducida para el precio del petróleo se puede expresar como:

$\mu_{\text {Lppetroleo }}=\varepsilon_{\text {Lppetroleo }}$

En [4] se muestra que el término error de la forma reducida del precio internacional del petróleo será igual al respectivo término error estructural. No obstante, en el caso de las otras variables del modelo, un cambio en el precio del petróleo tiene impacto sobre los costos de producción porque el petróleo es usado como un importante insumo en la producción y distribución de bienes y servicios.

En segundo lugar, se asume que la producción no responde de manera contemporánea (en el corto plazo) a cualquier cambio en las variables domésticas. En tercer lugar, se asume que el nivel de precios doméstico está influenciado por todas las variables, excepto por la tasa de cambio. Finalmente, no se imponen restricciones con respecto a la tasa de cambio, lo cual sugiere que esta responde a cambios en todas las variables. El término error de forma reducida de cada una de las variables domésticas (Lrpib, Lipc y Ltcr) se expresa así:

$\mu_{\text {Lppetroleo }}=\varepsilon_{\text {Lppetroleo }}$

$\mu_{\text {Lrpib }}=-a_{21} \mu_{\text {Lppetroleo }}+\varepsilon_{\text {Lrpib }}$

$\mu_{\text {Lipc }}=-a_{31} \mu_{\text {Lppetroleo }}=-a_{32} \mu_{\text {Lrpib }}+\varepsilon_{\text {Lrpib }}$

$\mu_{\text {Ltcr }}=-a_{41} \mu_{\text {Lppetroleo }}=-a_{42} \mu_{\text {Lrpib }}-a_{43} \mu_{\text {Lipc }}+\varepsilon_{\text {Ltcr }}$

La estimación del sistema [3] con las restricciones [4] a [6] permite medir la influencia de cambios en el precio del petróleo sobre el PIB, la inflación y la tasa de cambio real de los países que integran la AP. De acuerdo con el objetivo del estudio, este artículo centrará la atención sobre el efecto de las variaciones de los precios sobre el PIB.

Antes de estimar el modelo, se realizarán las correspondientes pruebas de estacionariedad, y luego de encontrar que las variables son $\mathrm{I}(1)^{23}$, se empleará la prueba de cointegración de Johansen (1988) con el fin de establecer la existencia y el número de relaciones de tendencias comunes entre las variables $L_{\text {ppetroleo }} t^{\prime} L_{\text {rpib }}{ }_{t^{\prime}} L_{i p c_{t}}$ y $L t c r_{t}$ dentro de cada uno de los países en estudio. Si se encuentra cointegración, se emplearán pruebas de causalidad de Granger y la función impulso-respuesta para determinar el efecto del precio del petróleo en las variables macroeconómicas empleadas.

\section{Datos}

Para estimar los correspondientes modelos VAR estructural, se emplearán datos trimestrales de 1980Q2 a 2014Q3 para el caso de Chile y México, de 1990Q1 a 2014Q3 para Perú y de 1994Q1 a

2 Con el fin de determinar el orden de integración de las series, se realizarán las siguientes pruebas: ADF (Dickey y Fuller, 1981), Phillips-Perron (Phillips y Perron, 1988), KPSS (Kwiatkowski et al., 1992), Breitung (Breitung, 2002), Zivot y Andrews (Zivot y Andrews, 1992) y HEGY (Hylleberg et al., 1990). 
2014Q3 para Colombia. Para cada país se usan el precio del petróleo, el PIB real, la inflación y la tasa de cambio real (TCR). Se utiliza el precio del crudo de petróleo West Texas Intermediate (WTI), que es considerado como el precio de referencia del precio mundial de petróleo y está medido en dólares estadounidenses. Las series de PIB real a precios constantes de 2010 y el índice de precios del consumidor para obtener la tasa de inflación de cada país son obtenidos de las Estadísticas Financieras Internacionales proporcionadas por el Fondo Monetario Internacional (FMI). Por su parte, la tasa de cambio real se calcula como el producto entre la tasa de cambio nominal (moneda local por cada dólar) y el índice de precios del consumidor de Estados Unidos, dividido por el índice de precios del consumidor del país, es decir, de cada uno de los países en estudio. Todas las variables están expresadas en forma de logaritmo.

\section{RESULTADOS EMPÍRICOS Y DISCUSIÓN}

En términos generales, una buena proporción de las variables macroeconómicas son no estacionarias, cuyo uso podría generar resultados espurios (Nelson y Plosser, 1982). Con el fin de analizar las estacionariedad de las series, se realizan las pruebas ADF (Dickey y Fuller, 1981), Phillips-Perron (Phillips y Perron, 1988), KPSS (Kwiatkowski et al., 1992), Breitung (Breitung, 2002) y Zivot y Andrews (Zivot y Andrews, 1992). Los resultados de las pruebas de raíces unitarias son reportados en la tabla 1.

En el caso del Lppetroleo, todas las pruebas concluyen que es una serie estacionaria en su primera diferencia, es decir, la serie es integrada de orden 1 o I(1). En Chile, el Lrpib es I(1) empleando las pruebas ADF, PP y Breitung; adicionalmente, la variable Lipc se considerará como I(1), teniendo en cuenta que PP y Breitung lo concluyen; y la Lrpib es I(1) según todas las pruebas, excepto ADF. Para Colombia, todas las pruebas concluyen que el Lrpib es I(1); en el caso del Lipc, las pruebas son contradictorias, pero para efectos del estudio se supone I(1), y Lipc es I(1) según todas las pruebas.
En el caso de México, el Lrpib se supone I(1) de acuerdo con Breitung y Zivot \& Andrews; el Lipc es I(1) según todas las pruebas; en el caso de $L t c r$, solo la prueba de Breitung afirma que es I(1). Dada la robustez de esta prueba no paramétrica, se seguirá trabajando asumiendo que esta serie es I(1). En el caso de Perú, el Lrpib es I(1) según todas las pruebas, excepto PP; sin embargo, se supone aquí que es I(1); con respecto a Lipc las pruebas llegan a conclusiones contradictorias, pero una vez más se supone que es I(1) para efectos del estudio; finalmente, la Ltcr es I(1) según todas las pruebas. De esta manera, teniendo en cuenta los resultados de las pruebas de raíces unitarias, se puede concluir que hay evidencia para asumir que todas las series son I(1) en todos los países, para efectos de hacer análisis de cointegración.

Como lo refieren Alonso y Semaán (2010), cuando se trabaja con datos con una periodicidad mayor a la anual — datos trimestrales o mensuales-, es común encontrar comportamientos que se repiten en iguales periodos de los años, es decir, las series presentan estacionalidad. Esta estacionalidad requiere ser modelada, y una forma de identificar si una serie presenta raíces unitarias estacionales en los datos de frecuencia trimestral o mensual es empleando la prueba de HEGY (Hylleberg, Engle, Granger y Yoo, 1990), la cual permite determinar si una serie trimestral se debe integrar o no, y en caso de requerirlo, cómo se debe hacer (Alonso y Semaán, 2010).

En la tabla 2 se reportan los resultados de la prueba de HEGY para las variables Lrpib, Lipc y Ltcr en cada uno de los países en estudio y también para el Lppetroleo. De acuerdo con los resultados de la prueba, el Lppetroleo presenta una raíz unitaria no estacional. En el caso de Chile, la variable Lrpib presenta una raíz unitaria no estacional semestral, al igual que Lipc y Ltcr. En el caso de Colombia, las series Lrpib y Lipc presentan una raíz unitaria no estacional, en tanto Ltcr tiene una raíz unitaria anual. En México, las series Lipc y Ltcr no presentan raíces unitarias estacionales, pero Lrpib tiene una raíz unitaria semestral.

En el caso de Perú, la serie Lrpib presenta una raíz unitaria no estacional semestral anual, y Lipc 


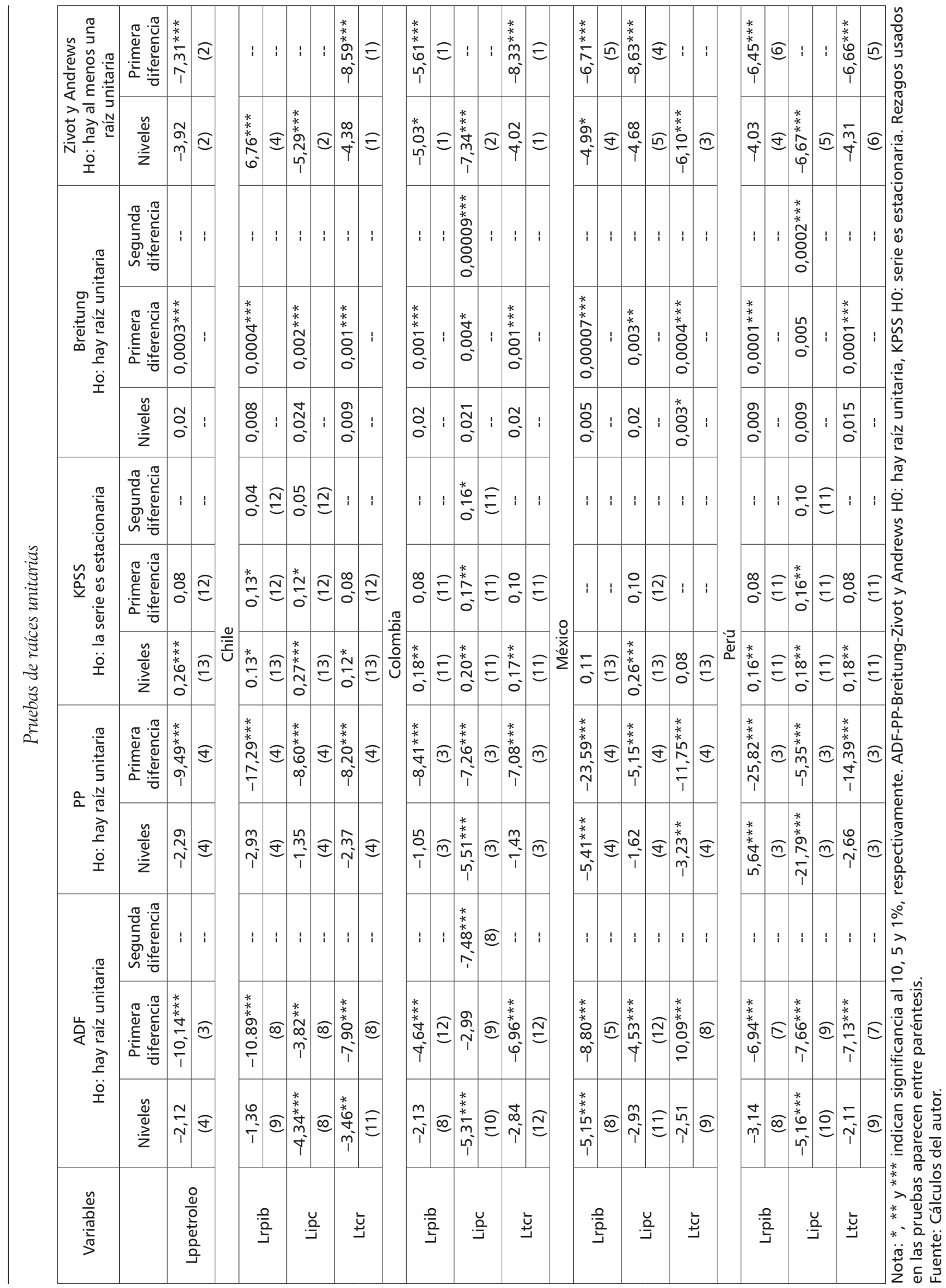


Tabla 2 .

Prueba de HEGY

\begin{tabular}{|c|c|c|c|c|c|c|c|c|c|c|c|c|c|}
\hline \multirow{2}{*}{$\begin{array}{l}\text { Hipótesis } \\
\text { nula }\end{array}$} & \multirow[b]{2}{*}{ Lppetroleo } & \multicolumn{3}{|c|}{ Chile } & \multicolumn{3}{|c|}{ Colombia } & \multicolumn{3}{|c|}{ México } & \multicolumn{3}{|c|}{ Perú } \\
\hline & & Lrpib & Lipc & Ltcr & Lrpib & Lipc & Ltcr & Lrpib & Lipc & Ltcr & Lrpib & Lipc & Ltcr \\
\hline$\pi_{1}=0$ & $-2,00$ & $-1,95$ & $-2,09$ & $-3,25$ & $-3,30$ & $-17,09$ & $-3,67^{* *}$ & $-3,94^{* *}$ & $-4,55^{* * *}$ & $-3,89^{* * *}$ & $-1,57$ & $-3,70^{* *}$ & $-1,68$ \\
\hline$\pi_{2}=0$ & $-9,49 * * *$ & $-0,68$ & $-4,55^{* * *}$ & $-5,39 * * *$ & $-3,98^{* * *}$ & $83,51 * * *$ & $-5,13 * * *$ & $-0,89$ & $-3,25^{* * *}$ & $-9,09 * * *$ & $-0,43$ & $-6,11 * * *$ & $-6,91 * * *$ \\
\hline$\pi_{3}=\pi_{4}=0$ & $36,20^{* * *}$ & $3,75^{* *}$ & $17,12^{* * *}$ & $10,74^{* * *}$ & $14,43^{* * *}$ & 0,97 & 1,67 & $3,01^{* *}$ & ** & 60, & 1,01 & $20,16^{* * *}$ & $60,70^{* * *}$ \\
\hline$\pi_{2}=\pi_{4}=0$ & $133,63^{* * *}$ & $2,67^{* * *}$ & $18,16^{* * *}$ & $22,49 * * *$ & $14,27^{* * *}$ & $277,43^{* * *}$ & $36,07^{* * *}$ & $2,25^{* * *}$ & $5,73^{* * *}$ & $147,89 * * *$ & 0,73 & $80,37^{* * *}$ & $93,19 * * *$ \\
\hline$\pi_{1}=\pi_{4}=0$ & $112,78^{* * *}$ & $2,99 * * *$ & $15,31^{* * *}$ & $20,00^{* * *}$ & $14,30^{* * *}$ & $218,49^{* * *}$ & $37,85^{* * *}$ & $5,85^{* * *}$ & $9,36^{* * *}$ & $110,94 * * *$ & 1,21 & $99,87^{* * *}$ & $71,81^{* * *}$ \\
\hline
\end{tabular}

Nota: *,** $y * * *$ indican significancia al 10,5 y $1 \%$, respectivamente.

Fuente: Cálculos del autor.

no presenta raíces unitarias estacionales; sin embargo, Ltcr presenta una raíz unitaria no estacional (ver apéndice 1, tablas 6 y 7). En línea con Alonso y Semaán (2010), las series Lrpib en Chile, Lipc en Colombia y Lrpib en Perú se pueden transformar usando polinomios del operador de rezagos para obtener series que solo tengan una raíz unitaria convencional, y no las estacionales, como se describe en la tabla 7 en el apéndice 1 de este artículo. Así, la prueba de cointegración de Johansen (1988), causalidad de Granger y análisis de descomposición de varianzas se realizan usando las series que no representan raíces unitarias estacionales.

Después de establecer las propiedades de estacionariedad de los datos, se procede a examinar las relaciones de largo plazo entre variables Lppetroleo, Lrpib, lipc y Ltcr en cada uno de los países en estudio, a través de la prueba de conintegración de Johansen (1988). La longitud de los rezagos VAR es determinado por el criterio de AIC, con lo cual se asegura que los errores del VAR estén libres de autocorrelación.

Los resultados de la prueba de cointegración de Johansen (1988) se presentan en la tabla 3. Con un nivel de significancia del 5\%, la hipótesis nula de no cointegración es rechazada; incluso, ambos estadísticos $\lambda$-trace y $\lambda$-max indican la presencia de dos vectores de cointegración ${ }^{3}$, es decir, $r=2$,

3 La existencia de vectores de cointegración implica que hay relación lineal de largo plazo entre las variables. entre las variables Lppetroleo, Lrpib, Lipc y Ltcr dentro de cada uno de los países de la AP. Esto implica que para cada uno de los países en estudio se presentan dos vectores de cointegración entre las variables. Estos resultados indican además que las variables macroeconómicas incluidas en este estudio para cada uno de los países que integran la AP no se mueven de manera independiente por un largo periodo. En otras palabras, las variables consideradas se mueven juntas en el largo plazo.

Tabla 3.

Prueba de cointegración de Johansen para Lppetroleo, Lrpib, Lipc y Ltcr en cada país

\begin{tabular}{|c|c|c|c|}
\hline País & Hipótesis nula & $\lambda$-trace & $\lambda$-max \\
\hline \multirow{4}{*}{ Chile } & $\mathrm{r}<=1$ & $34,52^{* *}$ & $21,84^{* *}$ \\
\cline { 2 - 4 } & $\mathrm{r}<=2$ & 12,68 & 10,70 \\
\hline \multirow{4}{*}{ Colombia } & $\mathrm{r}<=3$ & 1,98 & 1,98 \\
& $\mathrm{r}=0$ & $104,72^{* * *}$ & $57,42^{* * *}$ \\
\hline \multirow{4}{*}{ México } & $\mathrm{r}<=1$ & $47,30^{* * *}$ & $32,32^{* * *}$ \\
\cline { 2 - 4 } & $\mathrm{r}<=2$ & 14,98 & $14,68^{*}$ \\
\hline \multirow{3}{*}{ Perú } & $\mathrm{r}<=3$ & 0,30 & 0,30 \\
\cline { 2 - 4 } & $\mathrm{r}<=1$ & $38,65^{* * *}$ & $20,86^{*}$ \\
& $\mathrm{r}<=2$ & $17,79^{*}$ & 15,67 \\
\hline & $\mathrm{r}<=1$ & 2,12 & 2,12 \\
\hline & $\mathrm{r}<=2$ & $37,96^{* * *}$ & $27,46^{* * *}$ \\
& $\mathrm{r}<=3$ & 10,50 & 10,40 \\
\hline
\end{tabular}

Nota: ${ }^{*},{ }^{* *} y{ }^{* *}$ indican significancia al $10 \%, 5 \%$ y $1 \%$, respectivamente.

Fuente: Cálculos del autor. 


\section{Causalidad de Granger: causalidad de largo plazo y causalidad instantánea}

En las tablas 4 y 5 se reportan los resultados la prueba de causalidad de Granger. En la tabla 4, con un nivel de confianza del $99 \%$, la hipótesis nula según la cual el Lppetroleo no causa las variables macroeconómicas (Lrpib, Lipc y Ltcr) es rechazada en Chile, México y Perú. Este resultado permite afirmar que, en términos estadísticos, el Lppetroleo causa a lo Granger las variables macroeconómicas de estos países en el largo plazo. En Colombia, la hipótesis nula no se puede rechazar.

Por su parte, de acuerdo con la tabla 5, con un nivel de confianza del $99 \%$, la hipótesis nula de causalidad instantánea de Lppetroleo a variables macroeconómicas de los países en estudio es rechazada en el caso de Chile y Colombia, pero no se rechaza en el caso de México y Perú. De esta manera, es posible decir que cambios en Lppetroleo causan en el corto plazo las variables macroeconómicas de Chile y Colombia. Así, en general, los cambios en el Lppetroleo causan las variables macroeconómicas de Chile en el corto y largo plazo, de México y Perú solo en el largo plazo y de Colombia solo en el corto plazo.

\section{Funciones impulso-respuesta}

Con el fin de analizar el efecto de cambios inesperados en el precio del petróleo sobre el PIB, se calculan las funciones impulso-respuesta ante un cambio en un periodo del precio del petróleo sobre el Lrpib en cada uno de los países de la AP. Además, se estiman los respectivos intervalos de confianza por medio de Bootstraping, empleando 10.000 repeticiones. Los resultados de las funciones de impulso-respuesta son reportados en las Figuras 1, 2, 3 y 4 para Chile, Colombia, México y Perú, respectivamente. Cada figura muestra el efecto de un incremento inesperado del precio del petróleo (en logaritmos) sobre el curso presente y futuro del

Tabla 4.

Causalidad de Granger de largo plazo

\begin{tabular}{|c|c|c|}
\hline País & Hipótesis nula & Prueba F \\
\hline Chile & Ho: Lppetroleo no causa a la Granger a Lrpib, Lipc $y$ Ltcr & $2,35^{* * *}$ \\
\hline Colombia & Ho: Lppetroleo no causa a la Granger a Lrpib, Lipc $y$ Ltcr & $1,87^{*}$ \\
\hline México & Ho: Lppetroleo no causa a la Granger a Lrpib, Lipc $y$ Ltcr & $3,39^{* * *}$ \\
\hline Perú & Ho: Lppetroleo no causa a la Granger a Lrpib, Lipc $y$ Ltcr & $2,92^{* * *}$ \\
\hline
\end{tabular}

Nota: * ${ }^{* *} \mathrm{y} * * *$ indican significancia al $10 \%, 5 \%$ y $1 \%$, respectivamente.

Fuente: Cálculos del autor.

Tabla 5.

Causalidad instantánea

\begin{tabular}{|c|c|c|}
\hline País & Hipótesis nula & Prueba chi-cuadrado \\
\hline Chile & Ho: Lppetroleo no causa de manera instantánea a Lrpib, Lipc y Ltcr & $19,29^{* * *}$ \\
\hline Colombia & Ho: Lppetroleo no causa de manera instantánea a Lrpib, Lipc y Ltcr & $16,06^{* * *}$ \\
\hline México & Ho: Lppetroleo no causa de manera instantánea a Lrpib, Lipc y Ltcr & 6,24 \\
\hline Perú & Ho: Lppetroleo no causa de manera instantánea a Lrpib, Lipc y Ltcr & 3,94 \\
\hline
\end{tabular}

Nota: * ** $y * * *$ indican significancia al $10 \%, 5 \%$ y $1 \%$, respectivamente.

Fuente: Cálculos del autor. 
PIB en cada uno de los países de la AP. El intervalo de confianza reportado es al nivel del $95 \%$.

Figura 1.

Respuesta del PIB de Chile frente a un choque positivo e inesperado en el precio internacional del petróleo

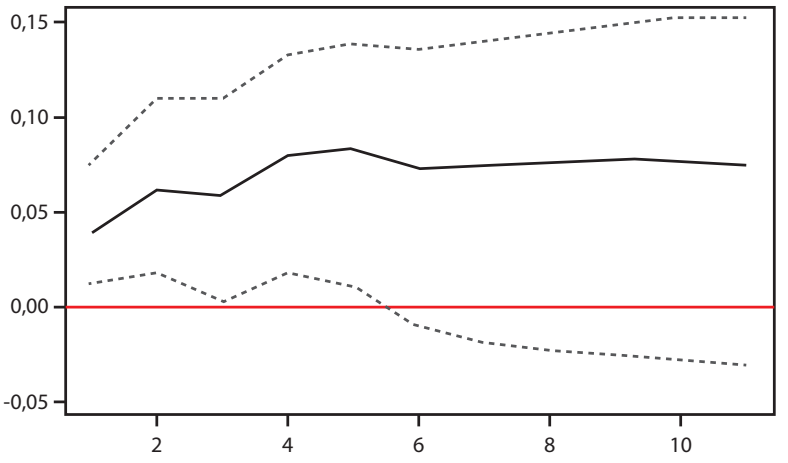

Fuente: Cálculos del autor.

La figura 1 muestra la respuesta de la producción ante un aumento inesperado en el precio del petróleo. La producción de Chile evidencia una respuesta positiva y estadísticamente significativa ante el choque positivo en el precio para los primeros cinco trimestres. Después del sexto trimestre, el efecto no es significativo. En otras palabras, el aumento del precio del petróleo genera un aumento en el nivel de actividad económica por un año y un trimestre. En este sentido, Basneta y Upadhyayab (2015) sostienen que el efecto de un incremento en el precio del petróleo es diferente para países importadores y exportadores de petróleo. Sugieren también que los países exportadores pueden experimentar un impacto positivo sobre la economía, al menos en el corto plazo. Este impacto positivo puede desvanecerse en el tiempo debido, entre otras razones, a mayores demandas salariales, lo cual presiona los costos de producción y precios del consumidor. Si bien Chile no es exportador de petróleo, el efecto del incremento en el precio del petróleo en la producción es positivo y contradice lo encontrado y sugerido por Basneta y Upadhyayab (2015).

La figura 2 presenta la respuesta de PIB de Colombia ante una innovación en el precio del petróleo. El efecto sobre la producción es positivo y significativo para los primeros seis trimestres. A partir de un año y medio (siete semestres), el choque inesperado en el precio del petróleo no tiene efecto significativo en el nivel de actividad económica en Colombia. Este resultado es consistente con los resultados encontrados en la literatura para países exportadores.

Figura 2.

Respuesta del PIB en Colombia frente a un choque positivo e inesperado en el precio internacional del petróleo

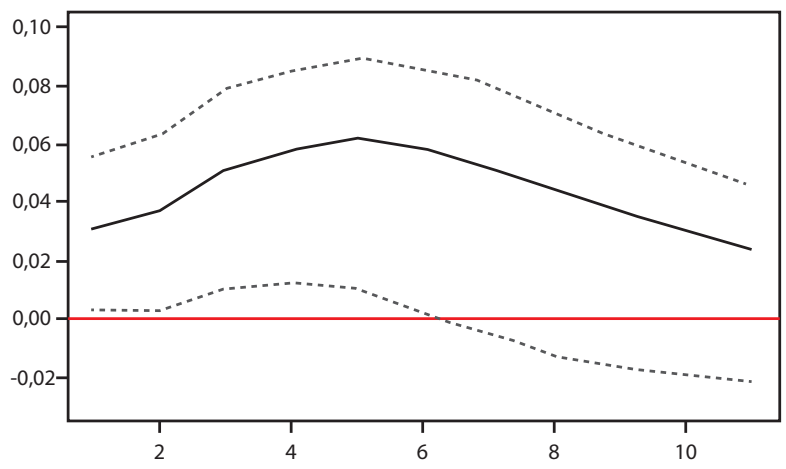

Fuente: Cálculos del autor.

La figura 3 muestra la respuesta del PIB de México ante el incremento inesperado en el precio del petróleo. Para el primer trimestre no existe efecto sobre el nivel de actividad económica. El efecto sobre la producción es positiva y significativa para el segundo y tercer trimestres, pero después el efecto no es significativo. Al igual que en el caso colombiano, la respuesta del nivel de actividad económica en México concuerda con lo encontrado en la literatura para países exportadores de petróleo.

Figura 3.

Respuesta del PIB en México frente a un choque positivo e inesperado en el precio internacional del petróleo

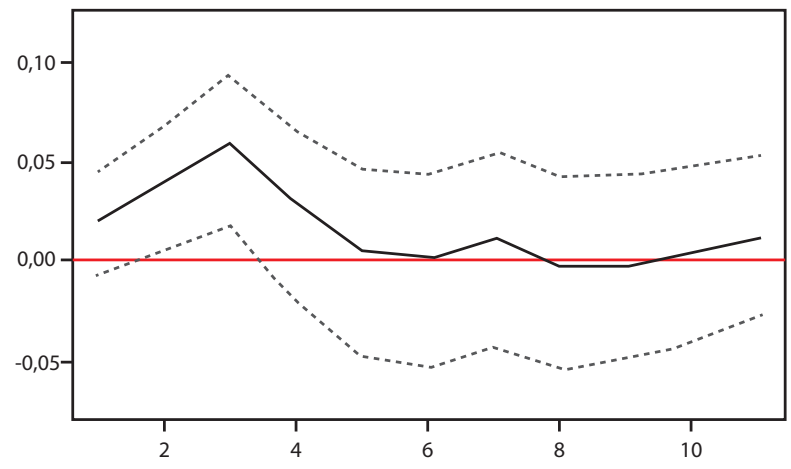

Fuente: Cálculos del autor. 
Finalmente, la figura 4 presenta la respuesta del nivel de actividad económica de Perú ante un aumento inesperado en el precio del petróleo. A diferencia de los otros tres países miembro de la Alianza del Pacífico, el PIB no se ve afectado por el choque en los precios del petróleo. Este resultado no concuerda con lo encontrado en la literatura para países importadores netos de petróleo.

Figura 4.

Respuesta de los agregados económicos en Perú frente a un choque positivo e inesperado en el precio internacional del petróleo

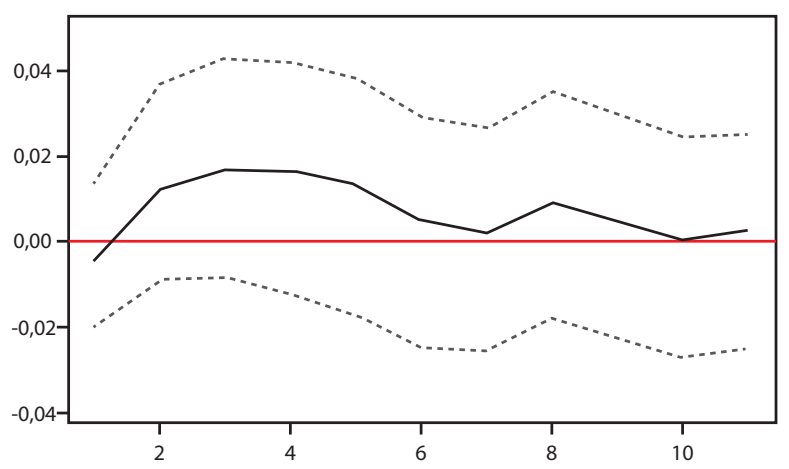

Fuente: cálculos del autor.

\section{COMENTARIOS FINALES}

El presente artículo examina de forma empírica el impacto de choques inesperados en el precio del petróleo sobre el PIB de cada uno de los países integrantes de la AP, usando un modelo VAR estructural. De igual manera, adopta una aproximación metodológica similar a la de Basnet y Upadhyaya (2015), quienes realizan un análisis similar para cinco de los países que integran la Asociación de Naciones del Sudeste Asiático (ASEAN, por sus siglas en inglés). Dichos autores encuentran que, en los cinco países, los efectos sobre el PIB de las variaciones en los precios del petróleo desaparecen después de cinco o seis trimestres.

En el caso de los países de la AP, la prueba de cointegración mostró que las variables macroeconómicas en estudio en cada país están cointegradas con el precio del petróleo, lo cual sugiere que en cada país el precio del petróleo, el PIB real, el índice de precios al consumidor y la tasa de cambio real tienen una relación de largo plazo.

En general, se encuentra que para Perú no existe relación alguna entre el PIB y los choques inesperados en el precio del petróleo. Este resultado no concuerda con lo se esperaría para un país que importa petróleo; por ende, ello amerita mayor investigación.

Otro resultado que no concuerda con lo esperado es el encontrado para Chile, país que es importador neto: el aumento inesperado en el precio del petróleo, si bien no tiene un efecto de largo plazo, sí genera un aumento en el PIB en el corto plazo (cinco trimestres). Este resultado podría explicarse por la gran dependencia de la economía chilena a la exportación de otros commodities de origen minero como el Cobre. Típicamente, la dinámica del precio de estos commodities tiene cierta similitud con el comportamiento del precio del petróleo. No obstante, esta explicación deberá ser probada empíricamente en futuras investigaciones.

Los resultados para los dos países exportadores de petróleo de la AP concuerdan en parte con lo esperado teóricamente. No obstante, el crecimiento inesperado de los precios del petróleo no tiene un efecto de largo plazo sobre estas dos economías. Esto es similar a lo encontrado por Basnet y Upadhyaya (2015) para Tailandia, Malasia, Singapur y Filipinas. Para México el efecto es menor: solo en el segundo y tercer trimestre se presenta el efecto sobre el PIB. En Colombia se presenta el efecto más prolongado en el tiempo en seis trimestres (año y medio).

Lo encontrado por este estudio permite plantear algunas implicaciones de política para los países que integran la AP. La coordinación de políticas entre los cuatro países ante choques externos como el aumento del precio del petróleo no será una tarea fácil. Las cuatro economías responden de manera diferente. Si bien México, Colombia y Chile tienen comportamientos similares, el tiempo de respuesta de las variables es muy diferente. Este es un buen ejemplo de los retos de coordinación que experimentarán las autoridades económicas en el proceso de armonización de las cuatro economías 
que conforman la AP. Así, uno de los aportes de este artículo es mostrar el gran reto de política macroeconómica que implicará armonizar las instituciones económicas y las respuestas de estas ante cambios en el ambiente macroeconómico.

Por otro lado, el presente modelo implica simetría en la respuesta del PIB ante cambios en el precio del petróleo; por lo tanto, la caída en el precio del petróleo experimentada durante 2015 y 2016, de acuerdo con nuestros resultados, tiene un efecto negativo sobre el nivel de actividad económica de Chile, México y Colombia. En especial, el efecto es más prolongado sobre el PIB en Colombia.

Finalmente, si bien los resultados de este estudio llaman la atención sobre las diferentes formas como reaccionan las economías ante un choque externo, aún hay mucho por investigar; por ejemplo, es importante determinar si el efecto de este tipo de choques sobre las variables macroeconómicas es simétrico o no.

\section{REFERENCIAS}

1. Alarco, G. (2006). La evolución del precio del petróleo crudo y la economía de México, 1975-2004. Comercio Exterior, 56(9), 930-944.

2. Alonso, J. C., y Semaán, P. (2010). Prueba de HEGY. Apuntes de Economía, 23, 1-28.

3. Backus, D. K., y Crucini, M. J. (2000). Oil prices and the terms of trade. Journal of International Economics, $50(1), 185-213$.

4. Basnet, H. C., y Upadhyaya, K. P. (2015). Impact of oil price shocks on output, inflation and the real exchange rate: evidence from selected ASEAN countries. Applied Economics, 47, 3078-3091.

5. Bernanke, B. (1983). Irreversibility, uncertainty, and cyclical investment. Quarterly Journal of Economics, 98(1), 85-106.

6. Breitung, J. (2002). Nonparametric tests for unit roots and cointegration. Journal of Econometrics, 108(2), 343-363.

7. Brown, S. P. A., y Yücel, M. K. (2002). Energy prices and aggregate economic activity: an interpretative survey. The Quarterly Review of Economics and Finance, 42(2), 193-208.

8. Cebula, R., Saadatmand, Y. y Piccone, Y. (2002). An empirical note on the inflation impact of the price of imported crude oil: The case of Germany. Rivista Internazionale di Scienze Economiche e Commerciali, 49(4), 531-537.

9. Christiano, L. J., Eichenbaum, M., y Vigfusson, R. (2007). Assessing structural vars. NBER Macroeconomics Annual, 21, 1-106).

10. Cologni, A., y Manera, M. (2008). Oil prices, inflation and interest rates in a structural cointegrated \{VAR\} model for the G-7 countries. Energy Economics, 30(3), 856-888.

11. Cunado, J., y de Gracia, F. P. (2005). Oil prices, economic activity and inflation: evidence for some Asian countries. The Quarterly Review of Economics and Finance, 45(1), 65-83.

12. Denis, D., y Etornam, D. K. (2015). Granger causality analysis on Ghana's macro-economic performance and oil price fluctuations. Journal of Resources Development and Management, 6, 1-5.

13. Dickey, D. A., y Fuller, W. A. (1981). Likelihood ratio statistics for autoregressive time series with a unit root. Econometrica, 49(4), 1057-1072.

14. Enders, W. (2004). Applied econometric time series. Wiley series in probability and mathematical statistics (2. ed.). Hoboken: J. Wiley. 
15. Farzanegan, M. R. y Markwardt, G. (2009). The effects of oil price shocks on the Iranian economy. Energy Economics, 31(1), 134-151.

16. Fernald, J. G., y Trehan, B. (2005). Why hasn't the jump in oil prices led to a recession? FRBSF Economic Letter. San Francisco: Federal Reserve Bank of San Francisco.

17. Gallardo, J., Vásquez Cordano, A., y Bendezú, L. (2005). La problemática de los precios de los combustibles. Recuperado de http://econpapers.repec.org/RePEc:ose:wpaper:11

18. Hamilton, J. D. (1983). Oil and the macroeconomy since World War II. Journal of Political Economy, 91(2), 228-248.

19. Hamilton, J. D. (1996). This is what happened to the oil price-macroeconomy relationship. Journal of Monetary Economics, 38(2), 215-220.

20. Hamilton, J. D. (2003). What is an oil shock? Journal of Econometrics, 113(2), 363-398.

21. He, Y., Wang, S., y Lai, K. K. (2010). Global economic activity and crude oil prices: A cointegration analysis. Energy Economics, 32(4), 868-876.

22. Hooker, M. A. (1996). What happened to the oil price-macroeconomy relationship? Journal of Monetary Economics, 38(2), 195-213.

23. Hooker, M. A. (1999). Oil and the Macroeconomy Revisited. Recuperado de http://dx.doi.org/10.2139/ ssrn. 186014

24. Huntington, H. G. (1998). Crude Oil Prices and U.S. Economic Performance: Where does the asymmetry reside? The Energy Journal, 19(4), 107-132.

25. Hylleberg, S., Engle, R., Granger, C. W. J. y Yoo, B. S. (1990). Seasonal integration and cointegration. Journal of Econometrics, 44, 215-238.

26. Jiménez-Rodríguez, R., y Sánchez, M. (2005). Oil price shocks and real GDP growth: empirical evidence for some OECD countries. Applied Economics, 37(2), 201-228.

27. Johansen, S. (1988). Statistical analysis of cointegration factors. Journal of Economic Dynamics and Control, $12(2-3), 231-254$.

28. Kilian, L. (2008). Exogenous oil supply shocks: How big are they and how much do They Matter for the U.S. Economy? Review of Economics and Statistics, 90(2), 216-240.

29. Kwiatkowski, D., Phillips, P. C. B., Schmidt, P., y Shin, Y. (1992). Testing the null hypothesis of stationarity against the alternative of a unit root : How sure are we that economic time series have a unit root? Journal of Econometrics, 54(1-3), 159-178.

30. Lardic, S., y Mignon, V. (2008). Oil prices and economic activity: An asymmetric cointegration approach. Energy Economics, 30(3), 847-855.

31. Mork, K. A. y Hall, R. E. (1980). Energy prices, inflation, and recession, 1974-1975. The Energy Journal, $1(3), 31-63$.

32. Nelson, C. R., y Plosser, C. R. (1982). Trends and random walks in macroeconmic time series. Journal of Monetary Economics, 10(2), 139-162.

33. Park, C., Chung, M., y Lee, S. (2011). The effects of oil price on regional economies with different production structures: A case study from Korea using a structural \{VAR\} model. Energy Policy, 39(12), 8185-8195.

34. Pedersen, M., y Ricaurte, M. (2013). Efectos de shocks al precio del petróleo sobre la economía de Chile y sus socios comerciales. Recuperado de http://vox.lacea.org/?q=precio_petroleo_chile 
35. Perilla, J. R. (2010). El impacto de los precios del petróleo sobre el crecimiento económico en Colombia. Revista de Economía del Rosario, 13(1), 75-116.

36. Phillips, P. C. B., y Perron, P. (1988). Testing for a unit root in time series regression. Biometrika, 75(2), 335-346.

37. Sill, K. (2007). The macroeconomics of oil shocks. Federal Reserve Bank of Philadelphia, Business Review, $1(1), 21-31$.

38. Zaidi, M. A. S., Karim, Z. A., y Azman-Saini, W. N. W. (2013). Foreign and domestic shocks: Macroeconomic responses of ASEAN-3 Countries. Global Economic Review, 42(3), 215-237.

39. Zhang, W., y Qianqian, Z. (2011). The Impact of international oil price fluctuation on China's Economy. Energy Procedia, 5, 1360-1364.

40. Zivot, E., y Andrews, D. W. K. (1992). Further evidence on the great crash, the oil-price shock, and the unit-root hypothesis. Journal of Business and Economic Statistics, 10(3), 251-270. 


\section{ANEXOS}

\section{Anexo 1}

Interpretación de los resultados de la prueba de HEGY

\begin{tabular}{|c|c|c|c|}
\hline $\begin{array}{c}\text { Hipótesis que NO se } \\
\text { rechazan }\end{array}$ & $\begin{array}{c}\text { Hipótesis que se } \\
\text { rechazan }\end{array}$ & Tipo de raíz unitaria presente & $\begin{array}{c}\text { Transformación para obtener variable } \\
\text { estacionaria }\end{array}$ \\
\hline$H_{A^{\prime}} H_{B^{\prime}} H_{C}$ & - & No estacional semestral anual & $\Delta 4 y t=y t-y t-4$ \\
\hline$H_{A^{\prime}} H_{B}$ & $H_{C}$ & No estacional semestral & $\Delta 2 y t=y t-y t-2$ \\
\hline$H_{A^{\prime}} H_{C}$ & $H_{B}$ & No estacional anual & $\begin{array}{c}(1-L)\left(1+L^{2}\right) y t \\
y t-y t-1+y t-2-y t-3\end{array}$ \\
\hline$H_{B^{\prime}} H_{C}$ & $H_{A}$ & Semestral anual & $(1+L)\left(1+L^{2}\right) y t$ \\
\hline$H_{A}$ & $H_{B^{\prime}} H_{C}$ & No estacional & $y t-1+y t-2+y t-3$ \\
\hline$H_{B}$ & $H_{A^{\prime}} H_{C}$ & Semestral & $\Delta 1 y t=y t-y t-1$ \\
\hline$H_{C}$ & $H_{A^{\prime}} H_{B}$ & Anual & $(1+L) y t=y t+y t-1$ \\
\hline- & $H_{A^{\prime}} H_{B^{\prime}} H_{C}$ & No hay & $\left(1+L^{2}\right) y t=y t+y t-2$ \\
\hline
\end{tabular}

Fuente: Alonso y Semaán (2010, p. 10).

\section{Anexo 2}

Conclusiones según la prueba de HEGY y las transformaciones realizadas

\begin{tabular}{|c|c|c|c|}
\hline Variable & $\begin{array}{l}\text { Tipo de raíz unitaria } \\
\text { presente }\end{array}$ & Transformación para obtener serie estacionaria & $\begin{array}{c}\text { Transformación para } \\
\text { obtener serie I(1) }\end{array}$ \\
\hline Lppetroleo & No estacional & $(1-L)$ & -- \\
\hline \multicolumn{4}{|c|}{ Chile } \\
\hline Lrpib & No estacional semestral & $(1-L)(1+L)$ & \\
\hline Lipc & No estacional & $(1-L)$ & -- \\
\hline Ltcr & No estacional & $(1-L)$ & -- \\
\hline \multicolumn{4}{|c|}{ Colombia } \\
\hline Lrpib & No estacional & $(1-L)$ & \\
\hline Lipc & No estacional anual & $(1-L)(1+L 2)$ & $(1+L 2)$ \\
\hline Ltcr & Anual & $(1+L 2)$ & No se puede \\
\hline \multicolumn{4}{|c|}{ México } \\
\hline Lrpib & Semestral & $(1+L)$ & No se puede \\
\hline Lipc & Ninguna & $\mathrm{Na}$ & $\mathrm{Na}$ \\
\hline Ltcr & Ninguna & $\mathrm{Na}$ & $\mathrm{Na}$ \\
\hline \multicolumn{4}{|c|}{ Perú } \\
\hline Lrpib & No estacional semestral anual & $(1-L)(1+L)(1+L 2)$ & $\begin{array}{c}(1+\mathrm{L})(1+\mathrm{L} 2)=1+\mathrm{L}+\mathrm{L} 2 \\
+\mathrm{L} 3\end{array}$ \\
\hline Lipc & No estacional & $(1-L)$ & - \\
\hline Ltcr & Ninguna & $\mathrm{Na}$ & $\mathrm{Na}$ \\
\hline
\end{tabular}

Fuente: cálculos del autor a partir de Alonso y Semaán (2010). 